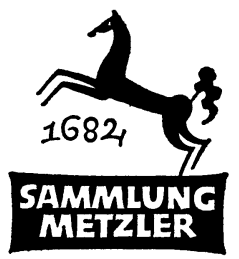

REALIENB UCHER FOR GERMANISTEN ABT. B: 
JOCHEN SCHULTE-SASSE

\section{Literarische Wertung}

MCMLXXI

J. B. METZLERSCHE VERLAGSBUCHHANDLUNG STUTTGART 
ISBN 978-3-476-10098-6

ISBN 978-3-476-99421-9 (eBook)

DOI 10.1007/978-3-476-99421-9

\section{98}

(C) 1971 Springer-Verlag GmbH Deutschland

Ursprünglich erschienen 1971 bei J. B. Metzlersche Verlagsbuchhandlung und Carl Ernst Poeschel Verlag GmbH in Stuttgart 


\section{VORWORT}

Zur literarischen Wertung können keine erkennbaren, zeitlos anwendbaren Fakten zusammengestellt werden wie z. B. zur Biographie eines Dichters. $\mathrm{Da}$ aber der Literaturwissenschaftler schon bei der Auswahl seiner Forschungsgegenstände in Wertungen verstrickt ist und es kaum ein Gebiet gibt, in dem sein Tun nicht durch Vor-Entscheidungen und Vor-Urteile bestimmt wäre, ist die Diskussion von Wertungsfragen ein wichtiger und notwendiger Bestandteil jeder wissenschaftstheoretischen Reflexion. Sie will literaturwissenschaftliche Urteile und Wertentscheidungen transparenter werden lassen.

In diesem Sinne will die Darstellung in eine lebendige und niemals abschließbare Diskussion einführen. Im Hauptteil sollen, der Zielsetzung der Reihe entsprechend, die vorherrschenden modernen Kitsch- und Wertungstheorien, deren Fronten heute immer noch recht verfestigt sind, referiert werden. Dieses Referat kann vom Gegenstand her nur sehr bedingt einen üblichen Forschungsbericht bieten und muß mit seinen Einwänden selbst im Kontinuum wissenschaftstheoretischer Reflexionen stehen. Deshalb wird die Darstellung weniger auf Erweiterungen, Vertiefungen oder bloße Variationen vorliegender Wertungstheorien eingehen als vielmehr das Gemeinsame herausstellen und - vor allem zum Schluß hin - die hermeneutischen Prämissen aktueller literarischer Werttheorien kritisch beleuchten und auf ihr erkenntnisleitendes Interesse zurückführen jedoch nicht mit dem Ziel, ein solches Interesse vorschnell als unwissenschaftlich $\mathrm{zu}$ verdammen. Die Literaturwissenschaft sollte ihr bildungs- und sozialgeschichtlich bedingtes und historisch gewachsenes Wertinteresse durchschauen lernen und sich zu ihm auf Grund zwar historisch vermittelter, aber ins Bewußtsein gehobener Wertentscheidungen bekennen, ohne dieses Wertinteresse, wie es in der deutschen Literaturwissenschaft so oft geschieht, als das ästhetisch, psychologisch und anthropologisch einzig mögliche und im Prinzip von der Geschichte unabhängige hinzustellen. In der Kraft der Selbstreflexion sollten, um eine Forderung von Jürgen Habermas aufzugreifen, Erkenntnis und Interesse der Literaturwissenschaftler eins werden. 
Eine knapp 80seitige Darstellung aktueller Kitsch- und Wertungstheorien, die annähernd 120 Veröffentlichungen zum Thema auf wenige vorherrschende und wirkungsmächtige Denkschemata zu reduzieren versucht, muß mit dem Einwand rechnen, sie habe wichtige eigenständige Diskussionsbeiträge nicht angemessen berücksichtigt. So ist z. B. Walther Killys Rückführung des Kitsches auf die Vorstellungswelt des Märchens, trotz zahlreicher Hinweise auf den Autor und seine Schrift über den Kitsch, nicht mit einem Wort erwähnt. In all diesen Fällen schien mir die Partizipation des jeweiligen Autors an den von mir beschriebenen Denkmodellen, vor allem im Hinblick auf die wissenschaftstheoretische Grundhaltung, wichtiger zu sein, als etwaige eigenständige, aber für die theoretischen Prämissen periphere Beiträge zur Kitsch- und Wertungsdiskussion. Daß so viele Schriften über den Kitsch und zur literarischen Wertung stereotyp einige, aus der klassisch-romantischen Tradition herzuleitende Denkfiguren wiederholen und gegen alle Forschungstradition nicht die Forschungsgeschichte rezipieren und rekapitulieren, hängt mit dem vorwissenschaftlichen Bekenntnischarakter der meisten werttheoretischen Schriften zusammen. Solange die Wertungstheorie Normen beschreibt, nach denen das literarische Bewußtsein in einer gegebenen historischen Situation transzendental arbeitet, sich mit diesen Normen identifiziert und sie nicht in ihrer sozial- und bildungsgeschichtlichen $\mathrm{Be}$ dingtheit untersucht und konsequent historisiert, solange beschreibt die paradoxe Formel von der „Wissenschaftlichkeit eines unwissenschaftlichen Themas" (Müller-Seidel) treffend den Stand der gegenwärtigen literaturwissenschaftlichen Wertungsdiskussion.

Für die Durchsicht des Manuskriptes und geduldige Hilfe beim Korrekturlesen danke ich Frl. Dr. Renate Werner, Frl. Dr. Marianne Schuller und Herrn stud. phil. Georg Behse.

Bochum

J. ScH.-S. 


\section{INHALTSVERZEICHNIS}

I. EINLETTENDE Hinweise . . . . . . . . . . . 1

1. Der Kitsch als Problem der literarischen Wertung ... 1

2. Nationale Besonderheiten der Wertungsprobleme ... 2

3. Wissenschaftsgeschichte der Werttheorien? . . . . . 6

II. Die Kontraposition Kunst - Kitsch . . . . . . . . . 9

1. Wertkriterien vorliegender Kitschtheorien . . . . . . 9 9

a. Architektonische und kumulative Strukturen . . . 10

b. Distanzierter und distanzloser Genuß . . . . . . 13

c. Geist und Sinnlichkeit . . . . . . . . . . 17

d. Geistige Beweglichkeit und Trägheit . . . . . . 18

e. Die "echtgeschöpfte Realitätsvokabel“ und das

Klischee . . . . . . . . . . . . . . . . . 19

2. Zur Geschichte, Relevanz und Gültigkeit des Kitschbegriffes . . . . . . . . . . . . . . . 22

a. Das klassisch-romantische Strukturmodell als normatives Leitbild und affirmatives Negativ der Kitschtheorien

b. Die übergeschichtliche Norm der Ganzheit. Erster Versuch einer Kritik . . . . . . . . . . . . 24

c. Wertkriterien als historisch gewachsene Denkzwänge

III. RANGORDNUNGSKRITERIEN FÜR DEN BEREICH, HOHER ${ }^{\circ}$

LITERATUR . . . . . . . . . . . . . . . . . . . 34

1. "Stimmigkeit" und „ästhetische Spannungen “ . . . . 34

2. Der mit künstlerischen Intentionen geschriebene, aber mißglückte Text: „Brüchigkeit" . . . . . . . . 40

3. Die Kontrapositionen . . . . . . . . . . . . . 43

Klischee - „echtgeschöpfte Realitätsvokabel“

gestaltet - geredet

echt - unecht/verlogen

ursprünglich - nicht-ursprünglich

Innovation - Redundanz

IV. Zu Funktion Und GÜLtigkeit MOdeRner WeRtungsTHEORIEN . . . . . . . . . . . . . . . 50

1. Von "metaphysischen " $\mathrm{zu}$,instrumentalen" Werttheorien ...............

a. Die metaphysischen Werttheorien der deutschen Literaturwissenschaft . . . . . . . . . . 
b. Zum geistesaristokratischen Erkenntnisinteresse der "Metaphysiker" . . . . . . . . . . 53

c. Zur Forderung nach, instrumentalen " Werttheorien 56

2. Zur Trennung von ästhetischer und außerästhetischer Wertung . . . . . . . . . . . . . . 59

3. Walter Müller-Seidels »Probleme der literarischen Wertung* - zeitgemäße Werttheorie auf „höherer Reflexionsstufe"? ............... . 64

4. Aufgaben einer literaturwissenschaftlichen Wertforschung 70

REGISTER . . . . . . . . . . . . . . . 75 


\section{AbKüRZUngen}

DU Der Deutschunterricht (Stuttgart)

DVjs. Deutsche Vierteljahrsschrift für Literaturwissenschaft und Geistesgeschichte

JA Johann Wolfgang Goethe: Sämtliche Werke. Jubiläumsausgabe, hrsg. von Eduard von der Hellen. Bd 1-40. 1902-1907

Lit. Problems of Literary Evaluation, ed. by

Evaluation Joseph Strelka. University Park/Md./USA u. London. 1969

OL Orbis Litterarum

WA Johann Wolfgang Goethe: Werke, hrsg. im Auftrage der Großherzogin Sophie von Sachsen-Weimar. Bd 1-143. 1887-1918. (Weimarer Ausgabe)

WW Wirkendes Wort

ZfdPh. Zeitschrift für dt. Philologie 